\title{
INNOVATIONS in pharmacy
}

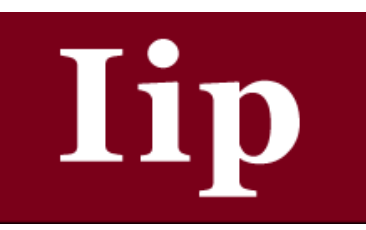

Volume 7 | Number 4

Article 15

$12-2-2016$

Evaluation of Qatari and Canadian Pharmacy Students Learning Together about Cultural Competency using Videoconference Educational Activities

Derek Jorgenson

University of Saskatchewan College of Pharmacy and Nutrition, derek.jorgenson@usask.ca

Kyle J. Wilby

Qatar University College of Pharmacy, kjw@qu.edu.qa

Jeff Taylor

University of Saskchewan College of Pharmacy and Nutrition, jeff.taylor@usask.ca

Follow this and additional works at: http://pubs.lib.umn.edu/innovations

\section{Recommended Citation}

Jorgenson D, Wilby KJ, Taylor J. Evaluation of Qatari and Canadian Pharmacy Students Learning Together about Cultural Competency using Videoconference Educational Activities. Inov Pharm. 2016;7(4): Article 15. http://pubs.lib.umn.edu/innovations/ vol7/iss $4 / 15$

INNOVATIONS in pharmacy is published by the University of Minnesota Libraries Publishing. 


\title{
Evaluation of Qatari and Canadian Pharmacy Students Learning Together about Cultural Competency using Videoconference Educational Activities
}

\author{
Derek Jorgenson, BSP, PharmD, FCSHP'; Kyle John Wilby, BSP, PharmD ${ }^{2}$; Jeff Taylor, BSP, PhD ${ }^{1}$ \\ ${ }^{1}$ University of Saskatchewan College of Pharmacy and Nutrition; ${ }^{2}$ Qatar University College of Pharmacy
}

\begin{abstract}
Background: Pharmacists are under pressure to provide patient centered care within increasingly culturally diverse settings. Pharmacy schools play an important role in educating learners regarding culture and its impact on patient care. Objectives: The objectives of this study were to determine if a novel cultural competency learning activity, which involved students from two culturally and ethnically different pharmacy schools learning together using videoconference education activities, improved: (1) student knowledge and confidence pertaining to cultural competency concepts, (2) attitudes and perceptions towards being a culturally competent pharmacist, and (3) academic performance related to cultural competency case studies. Methods: Pharmacy students from Qatar University in Doha, Qatar ( $n=25)$ and the University of Saskatchewan in Saskatoon, Canada ( $n=85)$ participated in a cultural competency activity comprised of small group work on a patient case study, followed by tutorial discussions. Some Canadian students $(n=31 / 85)$ worked collaboratively (via video conference) with the students from Qatar. The evaluation used a convergent mixed methods design comprised of: (1) a pre and post session survey measuring student knowledge and confidence; (2) pre and post session student self-reflections; and, (3) student academic performance on care plans and an observed structured clinical exam (OSCE). Results: The survey identified small but statistically significant $(p<0.05)$ improvements in knowledge and confidence with respect to 11 of the 12 questionnaire items in the students from Canada and 2 of the 12 items in the students from Qatar. The self-reflections found that 44.4\% (n=36/81) of students who completed the pre and post reflective questions reported a change in knowledge and attitudes regarding cultural competency, but a reason for the change was not evident. Student grades on the cultural competency care plans and the OSCE were not different between the students who worked collaboratively across the two schools compared with those who did not. Conclusion: The cultural competency student activity showed small improvements in pharmacy student knowledge, attitudes and confidence, but did not affect academic performance. Future research in this area could utilize a greater diversity of methodological approaches, including a focus on student self-reflection and qualitative assessment, to better capture student competency.
\end{abstract}

Key Words: culture, cultural competency, pharmacy education

\section{INTRODUCTION}

Populations in North America and abroad are becoming increasingly culturally diverse and significant health disparities exist between many groups. Health providers are being challenged to address these inequities through patient centered, culturally sensitive care. ${ }^{1,2}$ This underscores the importance of providing high-quality education for heath professional trainees regarding culture and its impact on patient care. $^{3}$

Cultural competence can be defined as the ability of health professionals to respectfully treat people of all cultures, ethnic backgrounds, and religions in a manner that recognizes, values and acknowledges their cultural differences in the care that is provided. ${ }^{3,4}$ The American College of Clinical Pharmacy (ACCP) published a White Paper on cultural competency in 2013, describing the critical need for pharmacy educators to incorporate knowledge and skills regarding cultural competency into undergraduate pharmacy curricula. ${ }^{3}$

Corresponding author: Dr. Derek Jorgenson, College of Pharmacy and Nutrition, University of Saskatchewan 104 Clinic Place, Saskatoon, Saskatchewan S7N 2Z4; P: 306-966-2009; F: 306-966-6656; Email: derek.jorgenson@usask.ca
Although pharmacy schools in many countries have begun to address the issue of incorporating culture into formal educational programming, nursing and medicine have made the greatest advances in this area. ${ }^{3,4}$ Nursing schools began integrating content regarding 'transcultural nursing' in the 1950's and cultural competency within medical schools is apparent in curricula dating back to the 1970's. ${ }^{3,4}$ The first official cultural diversity guideline for nursing education emerged in the late 1980's and both nursing and medical school accreditation bodies in the United States formally incorporated cultural competency requirements as early as 1999. 3,4

The movement to make cultural competency an integral part of pharmacy school curricula has only gained momentum within the last decade. ${ }^{3}$ The Accreditation Council for Pharmacy Education (ACPE) in the United States incorporated cultural competency into its 2006 (and subsequent) accreditation standards ${ }^{5}$ and the Association of Faculties of Pharmacy of Canada (AFPC) incorporated the concept into its education outcomes guideline in $2010 .^{6}$

The majority of research on the effectiveness of cultural competency curricular innovations in health professional training programs has focused on medical and nursing students. 
Beach et al. reviewed 34 articles in a systematic review on the topic, which reported that the educational interventions that have been evaluated are varied in both duration and instructional design, including: lectures, group discussions, cultural immersion, tours of hospitals and cultural communities, language lessons, written assignments, casebased learning, service learning, role play, and experiential education. ${ }^{4}$ Most of the studies in this systematic review demonstrated a small but measurable beneficial effect on learner knowledge and skills related to cultural competency; however, there is no clear pattern with respect to optimal instructional design. Beach et al. also reported that the evidence for the impact on learner attitudes is somewhat mixed, with some studies finding an improvement and others no effect. ${ }^{4}$ There is limited evidence that any of the interventions improve patient outcomes, aside from patient satisfaction. ${ }^{4}$

Reports of pharmacy school cultural competency educational programs include several short-term, single center studies evaluating a range of cultural competency curricular content. ${ }^{7-}$ ${ }^{15}$ Some studies found no impact, ${ }^{12,14,15}$ while others identified improvements in student knowledge or attitudes..$^{711,13}$ For example, in 2012, Haack et al. evaluated the impact of a series of cultural curricular changes, which included two hours of lectures and four hours of laboratory exercises, spaced across the first three years of the pharmacy program. ${ }^{12}$ The evaluation found no overall impact on the cultural competency knowledge or skills of students. In another study, Okoro et al. evaluated the impact of adding two lectures and two laboratory exercises to the existing curriculum. ${ }^{13}$ This study found an improvement in student knowledge related to health disparities and selfawareness, but no change in cultural competency skills. ${ }^{13}$

In an article reporting the results of four different studies, Hook et al. found that the development of cultural competency (or cultural humility) requires both intrapersonal and interpersonal components. ${ }^{16}$ Specifically, Hook et al. found that individuals must have an accurate view of themselves and their own cultural beliefs, while maintaining a stance focused on the 'other' (rather than self) characterized by mutual understanding and respect. These theoretical underpinnings endorse measurement techniques, such as reflection to measure student self-growth and actualization, when dealing with cultural conflict. ${ }^{17}$ As such, reflection can be prompted by case studies, standardized actors, or real-life patient encounters.

Despite the fact that pharmacy schools continue to strive to integrate cultural competency into their curricula, there is little guidance in the literature regarding optimal instructional design and assessment. The objectives of this study were to determine if a novel cultural competency learning activity, which was integrated simultaneously into two culturally diverse pharmacy schools, improves: (1) student knowledge and confidence pertaining to cultural competency concepts, (2) attitudes and perceptions towards being a culturally competent pharmacist, and (3) academic performance related to cultural competency case studies. The learning activity was a crossnational group work project in which pharmacy students from different countries worked together on an integrated case study that incorporated cultural components. This study was also innovative in its use of multiple methods to assess student academic performance.

\section{METHODS}

This study utilized a convergent mixed methods design in which quantitative and qualitative data were collected and analyzed to determine the impact on participants. The Behavioural Research Ethics Board at the University of Saskatchewan approved the protocol. Students were not required to provide consent to participate in the learning activity that was evaluated in this study, as it was a mandatory aspect of their academic programs. However, students were required to provide informed consent to have their assessment scores used for the analysis.

\section{Participants}

This study included all students enrolled (in September 2014) in their final professional year of the four-year bachelor of science in pharmacy program at the College of Pharmacy, University of Qatar $(n=25)$ and all students enrolled in the third year of the four-year bachelor of science in pharmacy program at the College of Pharmacy and Nutrition, University of Saskatchewan $(n=85)$. A staff member, who was not involved in assessing or evaluating the students approached them in person as a group during an existing laboratory session to request their informed consent to have their assessment scores used for the analysis.

These two schools were selected due to an existing collegial relationship between the researchers. The Canadian Council for Accreditation of Pharmacy Programs (CCAPP) accredits both programs, ensuring they meet similar standards with respect to content, quality and rigor. ${ }^{18}$ Students were recruited from different years within each program to ensure both groups had similar previous academic experiences with respect to the therapeutic topic of the curricular activity (e.g., asthma) and training related to developing care plans. Although both schools have similar academic programs accredited by CCAPP, the curricular content is delivered in a slightly different order in each program.

Despite the similarity of the curricular content at the two schools, they are different with respect to the cultural and ethnic backgrounds of the students enrolled. From an ethnicity perspective, $100 \%$ of the students from Qatar University originated from countries in the Middle East or North Africa, while $96.4 \%$ of students from the University of Saskatchewan 
originated from Canada. There was interest in whether the material would have different impact if implemented amongst two groups of students who have very different personal cultural and ethnic perspectives and experiences. It was hypothesized that teaching concepts and knowledge related to cultural competency might be more effective if participants from two pharmacy schools with culturally and ethically different students learned together, which is not something that has been previously studied in pharmacy.

\section{Description of the Curricular Activity}

To achieve the learning objectives, students were required to apply both their knowledge of pharmacotherapy and patient care skills to manage a patient case study, including identifying drug therapy problems and creating a patient centered care plan. The patient case study (Appendix 1) incorporated an aspect of the patient experience requiring a culturally sensitive approach by the pharmacist (e.g., a Saudi woman with asthma who was non-adherent to a corticosteroid for cultural-related beliefs), testing the student's ability to not only recognize this cultural issue, but to address it in their care plans.

At the beginning of October, small groups of students were established at each site. Approximately $1 / 3$ of the Canadian students ( $n=31 / 85$ ) were assigned to work in small groups with all of the 25 students from Qatar (i.e., 13 groups of 4-5 students were organized that included 2-3 students from each University). The remaining 54 Canadian students worked in similarly sized small groups without students from Qatar. Each group was given the same patient case study and were instructed to work within their small groups, outside of normal class time, to identify any drug therapy problems and create a care plan for the patient. The Qatar-Canada mixed groups were asked to work together to complete the task using any technology that they preferred to facilitate communication (e.g., video conference, text message, email), but a collaborative approach was mandatory for the creation of the care plans. The groups had three weeks during October to complete this task.

In early November, after completing the small group activity, students met in one of three large group, faculty-facilitated, tutorials that lasted approximately two hours. The goal of the tutorials was to provide the small groups with an opportunity to share and discuss the drug therapy problems that each group identified in the patient case, along with their care plans.

All of the Qatar-Canada mixed groups met during one tutorial using real-time videoconference technology, which was cofacilitated by faculty from both schools. The Canada-only small groups met in two separate tutorials facilitated by University of Saskatchewan faculty. Peer and faculty feedback was provided on all aspects of the case, including the culturally sensitive issue. No didactic teaching or additional curricular content was introduced during these tutorials. During all tutorials, the importance of cross-cultural communication and exploration of a patient's own beliefs regarding health and medications were emphasized during role-plays and large group discussions. These interventions aimed to develop students' understanding of cultural competency and ultimately influence their knowledge, attitude, and confidence pertaining to crosscultural interactions.

Student Knowledge and Confidence: Pre and post session survey A questionnaire designed to evaluate students' confidence and knowledge pertaining to cultural competency, using a 5-point Likert scale, was adapted from the published literature. ${ }^{8}$ The original questionnaire utilized by Muzumdar et al. included 29 questions relating to cultural competence knowledge and confidence, along with patient counselling skills and knowledge of alternative medicines. ${ }^{8}$ This study utilized only the Muzumdar article questions focused on cultural competency, resulting a questionnaire with 4 items related to cultural competency knowledge and 8 related to confidence (Appendix 2). Study investigators and one non-participant student in Qatar reviewed the 12 items in the questionnaire to ensure language and meaning applied to both contexts. English was used in both locations. The curriculum in Qatar is delivered in English and students are fluent with both spoken and written English.

In September (prior to implementation of the new learning activity), all participating students in Canada and Qatar were administered the questionnaire (paper-based) to determine pre-session confidence and knowledge relating to cultural competency. In March (after implementation of the new learning activity), students were administered the same questionnaire again to determine post-session scores. Questionnaires were administered during class time for an existing course in both schools. Results were entered into SPSS statistics (version 23.0, Amonk, NY: IBM Corp) and the presession scores were compared with post-session scores using an independent $t$-test. The survey instrument has not been validated to calculate an aggregate score for all combined items; therefore, pre and post session responses were compared for each individual item in the questionnaire.

The questionnaire that was used in both the September and March survey included a misprint that was not identified until after data had been collected. As can be noted in Appendix 2, the instructions for students at the top of the questionnaire stated that the scale ranged from 0 to 5 , when the actual scale used for each of the 12 questions ranged from 1 to 5 .

\section{Student Knowledge and Attitudes: Self Reflection Exercise}

In September, before the new learning activity occurred, all participating students in Canada and Qatar were asked to write a short ( $<250$ word) self-reflection to the following question: "What does it mean to be culturally competent as a 
pharmacist?" This reflection was meant to determine student's baseline knowledge and attitudes towards the concept of cultural competency. A reflective exercise was chosen. Reflection has been advocated as an ideal measure to assess student competency when faced with cultural conflict. ${ }^{17}$ Due its ability to stimulate self-awareness and personal growth, reflection helps promote students to recognize and acknowledge cultural conflict in interpersonal communication. ${ }^{17}$ Instructors can work with students to enrich the reflective process through evaluation of written content.

In March (after implementation of the new learning activity), participants had their original responses returned and were asked to respond to the following question: "Has anything changed with respect to your self-reflection, and if so, how and why have your views changed?"

The data from the self-reflection exercise was analyzed both quantitatively and qualitatively. Responses from all participants were entered into a Microsoft Excel for Mac 2011 (version 14.0, Redmond, WA: Microsoft Corp) spreadsheet and were analyzed using descriptive statistics. The free-text responses of participants who reported any sort of change between their initial self-reflection and their final self-reflection were analyzed using thematic analysis. All three authors independently reviewed the responses and categorized them into representative themes using open coding. ${ }^{19,20}$ After completing their independent analyses, the authors met once to confirm codes prior to creating themes. Authors met on a second occasion to discuss their final findings and come to consensus regarding a common set of themes.

\section{Student Academic Performance: Care Plan Grades}

The care plans developed by the small groups were graded with a standardized Global Ratings Scale rubric that is currently used at the University of Saskatchewan. For the purposes of this study, one additional domain was added to the existing care plan evaluation rubric, which allowed for faculty to assign a numeric score for the extent to which each group appropriately addressed cultural beliefs in their care plans (Appendix 3 ). This domain was added based on the goals of the tutorial and the underlying premise that cultural competency includes recognition of a patient's specific beliefs about health. ${ }^{21}$

The grades for the cultural competency domain of the care plan, along with the overall care plan grades were entered into SPSS statistics (version 23.0, Amonk, NY: IBM Corp) and the scores of the Qatar-Canada mixed student groups were compared with the scores of the Canada-only groups using an independent $t$-Test.
Student Academic Performance: Observed Structured Clinical Exam (OSCE) Grades

The existing curriculums of both schools include regular observed structured clinical exams (OSCE). Using existing exam development procedures at each school, a new OSCE station was developed, standardized and pre-tested. The investigators were directly involved in creating the new OSCE station, with the assistance of staff at each school, to ensure that the content of the station was directly related to the expected outcomes of the previous learning activities. The OSCE incorporated a patient issue that required a culturally sensitive approach (i.e., a patient who prefers to only take traditional / natural ethnic medicines, but who needs to be counseled regarding the need to take a $2^{\text {nd }}$ generation antihistamine) (Appendix 4). The patient issue that was used in the station was selected based on previous work using standardized patients to teach cultural competency to nurse practitioners. ${ }^{22}$ The final station underwent validation and piloting in both schools. This cultural competency OSCE station was included after the curricular activity was implemented in the regularly scheduled OSCE at each school (in December in Qatar and January in Canada). The OSCE evaluation rubric currently in use at Qatar University was used to assess student performance in the OSCE. This rubric generates a separate grade for patient centered communication, along with an overall grade (Appendix 4).

Communication and overall OSCE grades for the Canadian students were entered into SPSS statistics (version 23.0, Amonk, NY: IBM Corp) and the scores of the Canadian students who worked with the Qatar students were compared with the Canadian students who did not work with Qatar students, using an independent $t$-Test.

\section{RESULTS}

All students in both programs provided informed consent to allow their assessment scores to be used in the analysis of this study.

Student Knowledge and Confidence: Pre and Post Session Survey The anonymous pre-session questionnaire was completed by $84 / 85$ (98.8\%) of the students from Canada and 25/25 (100\%) of the students from Qatar. The post-session questionnaire was completed by $61 / 85$ (71.8\%) of the students from Canada and $24 / 25(96.0 \%)$ of the students from Qatar.

For the students from Canada, mean post-session questionnaire scores were statistically significantly higher for 11 of the 12 questionnaire items compared with the pre-session scores. For the students from Qatar, mean post-session scores were statistically significantly higher for 2 of the 12 questionnaire items (\#4 and \#8) (Table 1). 
Table 1: Mean Pre and Post Session Questionnaire Scores

\begin{tabular}{ccccccc}
\hline $\begin{array}{c}\text { Item } \\
\text { No. }\end{array}$ & $\begin{array}{c}\text { Canada } \\
\text { Students } \\
\text { Pre- } \\
\text { Session } \\
(\mathbf{n = 8 4 )}\end{array}$ & $\begin{array}{c}\text { Canada } \\
\text { Students } \\
\text { Post- } \\
\text { Session } \\
\text { (n=61) }\end{array}$ & $\begin{array}{c}\text { p- } \\
\text { value }\end{array}$ & $\begin{array}{c}\text { Qatar } \\
\text { Students } \\
\text { Pre- } \\
\text { session } \\
\text { (n=25) }\end{array}$ & $\begin{array}{c}\text { Qatar } \\
\text { Students } \\
\text { Post- } \\
\text { session } \\
(\mathbf{n = 2 4 )}\end{array}$ & $\begin{array}{c}\text { p- } \\
\text { value }\end{array}$ \\
\hline 1 & 3.43 & 4.02 & 0.01 & 4.20 & 4.38 & 0.39 \\
2 & 3.23 & 4.02 & 0.01 & 4.32 & 4.42 & 0.63 \\
3 & 3.60 & 3.85 & 0.08 & 3.76 & 4.17 & 0.14 \\
4 & 3.14 & 3.90 & 0.01 & 2.72 & 3.79 & 0.01 \\
5 & 2.93 & 3.59 & 0.01 & 3.64 & 3.92 & 0.27 \\
6 & 3.05 & 3.75 & 0.01 & 3.60 & 4.00 & 0.10 \\
7 & 3.29 & 3.85 & 0.01 & 3.72 & 4.04 & 0.15 \\
8 & 3.71 & 4.23 & 0.01 & 3.76 & 4.33 & 0.03 \\
9 & 3.29 & 3.93 & 0.01 & 3.76 & 4.13 & 0.09 \\
10 & 4.15 & 4.52 & 0.01 & 4.36 & 4.29 & 0.74 \\
11 & 3.82 & 4.38 & 0.01 & 3.76 & 4.21 & 0.08 \\
12 & 3.88 & 4.20 & 0.02 & 3.60 & 3.96 & 0.12 \\
\hline
\end{tabular}

Student Knowledge and Attitudes: Self Reflection Exercise The majority of students completed both pre and post session self-reflection exercises (Qatar students: $84.0 \%(n=21 / 25)$; Canadian students who worked alone: $75.9 \%$ ( $n=41 / 54)$; Canadian students who worked with Qatar students: $61.3 \%$ $(n=19 / 31))$. A large number of students in all three groups reported a change in their views (Table 2).

Table 2: Proportion of Students Reporting a Change in their Self-Reflection

\begin{tabular}{lcc}
\hline & $\begin{array}{c}\text { Reported a } \\
\text { change in self- } \\
\text { reflection (\%) }\end{array}$ & $\begin{array}{c}\text { Reported no } \\
\text { change in self- } \\
\text { reflection (\%) }\end{array}$ \\
\hline $\begin{array}{l}\text { Canadian students } \\
\text { (worked alone) } \\
(n=41)\end{array}$ & $15 / 41(36.6)$ & $26 / 41(63.4)$ \\
$\begin{array}{l}\text { Canadian students } \\
\text { (worked with }\end{array}$ & $8 / 19(42.1)$ & $11 / 19(57.9)$ \\
$\begin{array}{l}\text { Qatar students) } \\
\text { (n=19) }\end{array}$ & \\
$\begin{array}{l}\text { Qatar students } \\
(n=21)\end{array}$ & $13 / 21(61.9)$ & $8 / 21(38.1)$ \\
\hline
\end{tabular}

Only one theme emerged from the thematic analysis of the free-text responses of the students who reported a change in their self-reflection. Students reported that their knowledge and attitudes weren't really different, but they had "expanded". Most felt that they had a deeper and more thorough understanding of the concept of cultural competency at the end of the school year.
"I feel that my perception has not changed, but the depth of understanding of some cultural issues has expanded" (Canadian student)

"I don't think my answer has changed much, but I do think I've begun to understand what I wrote before on a more practical level. Before what I wrote seemed like a good answer in my head, it now feels like I've experienced some of those others ways of thinking." (Qatari student)

"Well I don't think my answer has changed much, but I do think I've begun to understand what I wrote on a more practical level." (Qatari student)

In addition, when responding to the second part of the opentext question ("How and why have your views changed?"), students did not provide any explanation for what caused their views to change. Notably, not a single student from either school mentioned the cultural competency intervention that was part of this study.

Student Academic Performance: Care Plan Grades

Student grades on the cultural competency domain of the care plan global rating scale were not statistically significantly different between the Canada-Qatar mixed groups and the Canada-only groups. Overall care plans grades were also not different between the two groups (Table 3).

Table 3: Care Plan Grades

\begin{tabular}{lccc}
\hline & $\begin{array}{c}\text { Qatari - } \\
\text { Canada } \\
\text { Mixed } \\
\text { Groups } \\
(\mathbf{n = 1 3 )}\end{array}$ & $\begin{array}{c}\text { Canada } \\
\text { Only } \\
\text { Groups } \\
(\mathbf{n = 1 4 )}\end{array}$ & p-value \\
\hline $\begin{array}{l}\text { Mean cultural } \\
\text { competency }\end{array}$ & $2.3 / 5.0(1.42)$ & $\begin{array}{c}2.8 / 5.0 \\
(1.12)\end{array}$ & 0.425 \\
$\begin{array}{l}\text { grade (SD) } \\
\text { Mean overall }\end{array}$ & $3.8 / 5.0$ & $3.6 / 5.0$ & 0.108 \\
$\begin{array}{l}\text { care plan } \\
\text { grade (SD) }\end{array}$ & $(0.584)$ & $(0.866)$ & \\
\hline
\end{tabular}

Student Academic Performance: Observed Structured Clinical Exam (OSCE) Grades

Student grades on the patient centred communication domain of the OSCE grading rubric were not statically significantly different between the Canadian-Qatar mixed groups and the Canada-only group. Overall OSCE exam grades were also not different between the two groups (Table 4). 
Table 4: OSCE Grades

\begin{tabular}{lccc}
\hline & $\begin{array}{c}\text { Canadian } \\
\text { students } \\
\text { who worked } \\
\text { with Qatar } \\
\text { students } \\
(\mathbf{n = 3 1 )}\end{array}$ & $\begin{array}{c}\text { Canadian } \\
\text { students } \\
\text { who did } \\
\text { not work } \\
\text { with Qatar } \\
\text { students } \\
(\mathbf{n = 5 4 )}\end{array}$ & p-value \\
\hline $\begin{array}{l}\text { Mean patient } \\
\text { centred }\end{array}$ & $77.2 \%(9.58)$ & $\begin{array}{c}75.6 \% \\
(9.78)\end{array}$ & 0.472 \\
$\begin{array}{l}\text { communication } \\
\text { grade (SD) }\end{array}$ & & & \\
$\begin{array}{l}\text { Mean overall } \\
\text { OSCE grade }\end{array}$ & $76.6 \%(7.89)$ & $76.3 \%$ & 0.880 \\
(SD) & & $(8.56)$ & \\
\hline
\end{tabular}

\section{DISCUSSION}

This study evaluated a collaborative case workup and tutorial completed locally or completed with participants from another country. In addition, it is the first cultural competency study in pharmacy students to evaluate the impact on student academic performance.

The results of this study suggest that the case workup and tutorial had a statistically significant impact on student knowledge, attitude and confidence related to cultural competency. However, the results also suggest that the learning activity did not affect student academic performance and the collaborative work between the students in Canada and Qatar likely did not enhance academic performance as assessed by care plan completion and performance in an OSCE.

The self-reflection data provide evidence that many students, regardless of which group they were in, developed deeper and expanded knowledge and attitudes regarding cultural competency by the end of the year. This is consistent with other studies that have implemented similar cultural competency student learning activities. ${ }^{9,11,12,14,15}$ Unfortunately, none of the students who reported a change in the self-reflections were able to determine what caused their views to change. It is certainly possible that the intervention in this study contributed to learning, but students did not consciously perceive the connection. Neither school had additional cultural competency activities scheduled during the study period; however, the student self-reflection results may have been influenced by other non-scheduled and informal cultural experiences encountered during the study period.

The lack of difference between the self-reflections and academic performance of students from Canada who worked with students from Qatar, and those who did not, raise doubt that the collaborative work between the students enhanced their learning. If our initial assumption was correct that learning would be enhanced if students from culturally and ethnically different backgrounds worked together, one would have expected a difference. However, it is possible that our sample was not large enough to detect this difference, or that our measurement tools were not sensitive enough to capture the changes. It is also possible that the case and/or group work need to be strengthened to better ensure meaningful dialogue and cross-participant learning.

Although data could not be interpreted by group, the results of the pre and post session survey provide some evidence that the learning activities had a positive impact on student knowledge and confidence. Participants from both schools had improved knowledge and confidence scores. The absolute size of the improvement was small (approximately 0.5 points on a 5 -point scale); however, these changes are similar to those reported when this questionnaire was used previously in the literature. ${ }^{8}$

It is difficult to interpret the apparent differences in survey scores between the students in Canada and Qatar. The students from Qatar appear to have higher baseline pre-session scores on some of the items, suggesting that the two groups may not have been similar at the start of the study with respect to cultural competency knowledge and confidence. This may be due to the fact that the students at Qatar University represent 8 different nationalities, while $96.4 \%$ of the University of Saskatchewan students originated from Canada.

The misprint that was included in the pre and post session survey, which referred to a scale from 0 to 5 in the student instructions, but actually used a scale from 1 to 5 , bring the trustworthiness of these data into question. However, the goal of the survey was not to analyze the meaning of the specific numbers that students selected, but rather to measure the change in their scores between September and March. Therefore, we believe that these results can still be used, along with the other measures reported in this study, to interpret the impact of the educational activity.

None of the previously published studies of cultural competency interventions within pharmacy schools evaluated student academic performance. ${ }^{7-15}$ The findings of this study provide the first evidence in the literature regarding the impact of a pharmacy school cultural competency intervention on student performance.

Based on our findings and experience, we can suggest recommendations for future studies attempting to evaluate academic performance relating to cultural competency. As a 'culturally diverse interaction' may be different for each individual, we strongly urge educators to use reflection and qualitative assessment methods to capture student competence. The use of a checklist, rubric and/or OSCE station may be helpful in assessing discrete cultural competency 
related skills, such as the ability to identify a culture related issue that may impact care. However, additional approaches are needed. For instance, future research could focus on the use of facilitated reflection exercises, ideally from real life patient encounters where the individual student recognized some form of cultural conflict. A diversity of approaches may be helpful in understanding the breadth of cultural competency related skills and also in understanding student growth. In addition, future research could evaluate modified assessment methods to better capture student competency.

The size of the study and the fact that it was based within only two pharmacy schools may limit the generalizability of the results. However, previous studies recruited similar numbers of participants, mostly within a single school. ${ }^{7-15}$ In addition, despite attempting to recruit students from two schools with similar curriculums, it appears, based on the results of the pre and post intervention survey, that the two groups may not have been equal at baseline with respect to their knowledge and confidence regarding cultural competency.

\section{CONCLUSION}

This study found that a cultural competency student learning activity comprised of small group work and large group tutorial discussions improved pharmacy student knowledge, attitudes and confidence related to cultural competency, but did not improve student academic performance. Future research in this area could utilize a greater diversity of methodological approaches, including a focus on student self-reflection and qualitative assessment, to better capture student competency.

\section{ACKNOWLEDGEMENTS}

The authors would like to acknowledge all of the pharmacy students at Qatar University and the University of Saskatchewan who participated in this study. Their enthusiasm and passion for learning was the inspiration for this work.

\section{DISCLOSURES}

None of the authors have any perceived or real conflicts of interest to report.

\section{REFERENCES}

1. Betancourt JR. Cross-cultural medical education: Conceptual approaches and frameworks for evaluation. Acad Med. 2003;78(6):560-569.

2. Shaya FT, Gbarayor CM. The case for cultural competence in health professions education. Am J Pharm Educ. 2006;70(6):Article 124.

3. American College of Clinical Pharmacy, O'Connell MB, Rodriguez de Bittner $\mathrm{M}$, et al. Cultural competency in health care and its implications for pharmacy part 3A: Emphasis on pharmacy education, curriculums, and future directions. Pharmacotherapy. 2013;33(12):e347-67.
4. Beach MC, Price EG, Gary TL, et al. Cultural competence: A systematic review of health care provider educational interventions. Med Care. 2005;43(4):356-373.

5. Accreditation Council for Pharmacy Education. Accreditation standards and gudelines. https://www.acpeaccredit.org/standards/default.asp. Updated 2016. Accessed May 12, 2016.

6. Association of Faculties of Pharmacy of Canada. Educational outcomes for first professional degree programs in pharmacy in canada.

http://www.afpc.info/node/39. Updated 2010. Accessed May 12, 2016.

7. Evans E. An elective course in cultural competence for healthcare professionals. Am J Pharm Educ. 2006;70(3):Article 55.

8. Muzumdar JM, Holiday-Goodman M, Black C, Powers M. Cultural competence knowledge and confidence after classroom activities. Am J Pharm Educ. 2010;74(8):Article 150.

9. Poirier TI, Butler LM, Devraj R, Gupchup GV, Santanello C, Lynch JC. A cultural competency course for pharmacy students. Am J Pharm Educ. 2009;73(5):Article 81.

10. Vyas D, Caligiuri FJ. Reinforcing cultural competency concepts during introductory pharmacy practice experiences. Am J Pharm Educ. 2010;74(7):Article 129.

11. Haack S. Engaging pharmacy students with diverse patient populations to improve cultural competence. Am J Pharm Educ. 2008;72(5):Article 124.

12. Haack $S$, Phillips $C$. Teaching cultural competency through a pharmacy skills and applications course series. Am J Pharm Educ. 2012;76(2):Article 27.

13. Okoro O, Odedina F, Smith WT. Determining the sufficiency of cultural competence instruction in pharmacy school curriculum. Am J Pharm Educ. 2015;79(4):Article 50.

14. Sales I, Jonkman L, Connor S, Hall D. A comparison of educational interventions to enhance cultural competency in pharmacy students. Am J Pharm Educ. 2013;77(4):Article 76.

15. Brown $B$, Heaton PC, Wall A. A service-learning elective to promote enhanced understanding of civic, cultural, and social issues and health disparities in pharmacy. Am J Pharm Educ. 2007;71(1):Article 9.

16. Hook JN, Davis DE, Owen J, Worthington EL, Utsey SO. Cultural humility: Measuring openness to culturally diverse clients. J Couns Psychol. 2013;60(3):353-366.

17. Rajaram S, Bockrath S. Cultural competence: New conceptual insights into its limits and potential for addressing health disparities. J Health Dispar Res Pract. 2014;7(5):Article 82. 
18. Canadian Council for Accreditation of Pharmacy Programs. Accreditation standards for the first professional degree in pharmacy programs.

http://ccapp-accredit.ca/. Updated 2013. Accessed May 12, 2016.

19. Crabtree BF, Miller WL, eds. Doing qualitative research. 2nd ed. Thousand Oaks, California: Sage Publications; 1999.

20. Boyatzis R, ed. Transforming qualitative information: Thematic analysis and code development. Thousand Oaks, California: Sage Publications; 1998.

21. Betancourt JR, Green AR, Carrillo JE, AnanehFirempong 02. Defining cultural competence: A practical framework for addressing racial/ethnic disparities in health and health care. Public Health Rep. 2003;118(4):293-302.

22. Rutledge CM, Garzon L, Scott M, Karlowicz K. Using standardized patients to teach and evaluate nurse practitioner students on cultural competency. Int J Nurs Educ Scholarsh. 2004;1:Article 17. 